\title{
Fractional-Order Accumulative Linear Time-Varying Parameters Discrete Grey Forecasting Model
}

\author{
Pumei Gao $\mathbb{D}^{1},{ }^{1}$ Jun Zhan, ${ }^{1,2}$ and Jiefang Liu $\mathbb{D}^{3}$ \\ ${ }^{1}$ School of Economics \& Management, Shanghai Maritime University, Shanghai 201306, China \\ ${ }^{2}$ School of Business Management, Shanghai Lixin University of Accounting and Finance, Shanghai 201209, China \\ ${ }^{3}$ School of Mathematical Science, Henan Institute of Science and Technology, Xinxiang 453003, China \\ Correspondence should be addressed to Pumei Gao; gaopm507@126.com
}

Received 27 February 2019; Revised 4 April 2019; Accepted 18 April 2019; Published 2 May 2019

Academic Editor: Ines Tejado Balsera

Copyright (C) 2019 Pumei Gao et al. This is an open access article distributed under the Creative Commons Attribution License, which permits unrestricted use, distribution, and reproduction in any medium, provided the original work is properly cited.

\begin{abstract}
Traditional discrete grey forecasting model can effectively predict the development trend of the stabilizing system. However, when the system has disturbance information, the prediction result will have larger error, and there will appear significant downward trend in the stability of the model. In the presence of disturbance information, this paper presents a fractional-order linear timevarying parameters discrete grey forecasting model to deal with the system that contains both linear trend and nonlinear trend. The modeling process of the model and calculation method are given. The perturbation bounds of the new model are analyzed by using the least-squares method of perturbation theory. And it is compared with that of the first-order linear time-varying parameters discrete grey forecasting model. Finally, two real cases are given to verify the effectiveness and practicality of the proposed method.
\end{abstract}

\section{Introduction}

The grey system theory was proposed by Deng in 1982. It only needs a small amount of data to establish the model for analysis $[1,2]$. Grey prediction model is the main part of grey system theory, and its theoretical basis is the grey of accumulation generation. The prediction model is established by using the characteristic of grey exponential. Since the grey prediction model was proposed, it has been widely concerned by scholars. The existing studies mainly focus on the theoretical development from the aspects of background value optimization [3, 4], parameter optimization [5-7], model expansion [8-10], and the application of natural gas [11, 12], electric power [13-16], environment [17-20], economy $[21,22]$, and transportation $[23,24]$.

Dang gave the new method to select initial value [25]. Cui proposed the NGM $(1,1, \mathrm{k})$ model and described the modeling mechanism and modeling process of the model [26]. Zhou proposed the generalized GM(1,1) model and used the new model to simulate and predict China's fuel output from 2003 to 2010 [27]. Combined with the concept of Bernoulli differential equation, Chen proposed the $\operatorname{NGBM}(1,1)$ model on the basis of GM(1,1) model [28]. Li proposed $3 \operatorname{spGM}(1,1)$ model and applied the new model to the failure data sets of electric product manufacturing systems [29]. These methods further improve the modeling effect of grey prediction model. However, the transformation of difference equation and differential equation is required in the solving process. There is still error in the grey prediction model for the sequences that conform to the exponential features.

Xie proposed the discrete grey model (DGM $(1,1)$ model). The relationship between $\operatorname{DGM}(1,1)$ and $\operatorname{GM}(1,1)$ was studied deeply, and the reason for the instability of $\operatorname{GM}(1,1)$ was found. It only needs to use difference equation to solve the equation and does not need to convert the difference equation to differential equation. Therefore, the modeling accuracy is effectively improved [30]. Wu proposed the discrete grey prediction model based on fractional-order accumulation, discussed the properties of the model, and gave the calculation method of the model [31-33]. Liu proposed the fractionalorder reverse accumulation discrete grey prediction model and discussed the properties of the model [34]. 
The research mentioned above has positive significance for improving the accuracy of grey prediction model. However, for complex systems with disturbance, the model's robustness is insufficient; how to deal with the system disturbance information is particularly important. To solve this problem, this paper presents a fractional-order accumulation linear time-varying parameters discrete grey prediction model (FTDGM(1,1)model). The modeling process and parametric calculation method of the model are given. It is proven that the model has good stability using the theory of matrix perturbation analysis. Finally, two real cases are given. And the calculation results show that FTDGM $(1,1)$ model can effectively reduce the disturbance caused by disturbance information. The model's robustness and prediction accuracy are improved, and the validity and practicality of the model are further verified.

\section{The Fractional-Order Accumulative Linear Time-Varying Parameters Discrete Grey Model}

Definition 1 (see [30]). Assume that the nonnegative sequence is $X^{(0)}=\left\{x^{(0)}(1), x^{(0)}(2), \cdots, x^{(0)}(n)\right\} . X^{(1)}=$ $\left\{x^{(1)}(1), x^{(1)}(2), \cdots, x^{(1)}(n)\right\}$ is the first-order accumulative sequence of $X^{(0)}$.

Among them,

$$
x^{(1)}(k)=\sum_{i=1}^{k} x^{(0)}(i), \quad k=1,2, \cdots, n .
$$

The equation

$$
x^{(1)}(k+1)=\beta_{1} x^{(1)}(k)+\beta_{2}, \quad k=1,2, \cdots, n-1
$$

is called discrete grey prediction model (DGM(1,1) model).

Theorem 2 (see [30]). The parameters of the DGM(1,1) model can be solved by using the following least-squares estimation:

$$
\left[\begin{array}{l}
\beta_{1} \\
\beta_{2}
\end{array}\right]=\left(B^{T} B\right)^{-1} B^{T} Y
$$

and, among them,

$$
\begin{aligned}
& B=\left[\begin{array}{cc}
x^{(1)}(1) & 1 \\
x^{(1)}(2) & 1 \\
\vdots & \vdots \\
x^{(1)}(n-2) & 1 \\
x^{(1)}(n-1) & 1
\end{array}\right], \\
& Y=\left[\begin{array}{c}
x^{(1)}(2) \\
x^{(1)}(3) \\
\vdots \\
x^{(1)}(n-1) \\
x^{(1)}(n)
\end{array}\right]
\end{aligned}
$$

Definition 3 (see [35]). Assume that the nonnegative sequence is $X^{(0)}=\left\{x^{(0)}(1), x^{(0)}(2), \cdots, x^{(0)}(n)\right\} . X^{(1)}=$ $\left\{x^{(1)}(1), x^{(1)}(2), \cdots, x^{(1)}(n)\right\}$ is the first-order accumulative sequence of $X^{(0)}$.

Among them,

$$
x^{(1)}(k)=\sum_{i=1}^{k} x^{(0)}(i), \quad k=1,2, \cdots, n .
$$

The equation

$$
\begin{aligned}
x^{(1)}(k+1)=\left(\beta_{1} k+\beta_{2}\right) x^{(1)}(k)+\beta_{3} k & +\beta_{4}, \\
& k=1,2, \cdots, n-1
\end{aligned}
$$

is called linear time-varying parameters discrete grey model (TDGM).

Theorem 4. The parameters of the TDGM(1,1) model can be solved by using the following least-squares estimation:

$$
\left[\begin{array}{l}
\beta_{1} \\
\beta_{2} \\
\beta_{3} \\
\beta_{4}
\end{array}\right]=\left(C^{T} C\right)^{-1} C^{T} Y
$$

and, among them,

$$
\begin{aligned}
& C=\left[\begin{array}{cccc}
x^{(1)}(1) & x^{(1)}(1) & 1 & 1 \\
2 x^{(1)}(2) & x^{(1)}(2) & 2 & 1 \\
\vdots & \vdots & \vdots & \vdots \\
(n-2) x^{(1)}(n-2) & x^{(1)}(n-2) & n-2 & 1 \\
(n-1) x^{(1)}(n-1) & x^{(1)}(n-1) & n-1 & 1
\end{array}\right], \\
& Y=\left[\begin{array}{c}
x^{(1)}(2) \\
x^{(1)}(3) \\
\vdots \\
x^{(1)}(n-1) \\
x^{(1)}(n)
\end{array}\right] .
\end{aligned}
$$

Definition 5 (see [18]). Assume that the nonnegative sequence is $X^{(0)}=\left\{x^{(0)}(1), x^{(0)}(2), \cdots, x^{(0)}(n)\right\}$.

$X^{(r)}=\left\{x^{(r)}(1), x^{(r)}(2), \cdots, x^{(r)}(n)\right\}$ is called the fractional-order accumulative sequence of $X^{(0)}$.

Among them,

$$
\begin{aligned}
& x^{(r)}(k)=\sum_{i=1}^{k} C_{k-i+r-1}^{k-i} x^{(0)}(i), \\
& \quad C_{r-1}^{0}=1, C_{k-1}^{k}=0, k=1,2, \cdots, n .
\end{aligned}
$$


Definition 6. Assume the nonnegative sequence $X^{(0)} ; X^{(r)}$ is defined as Definition 5. The equation

$$
\begin{array}{r}
x^{(r)}(k+1)=\left(\beta_{1} k+\beta_{2}\right) x^{(r)}(k)+\beta_{3} k+\beta_{4}, \\
\quad k=1,2, \cdots, n-1
\end{array}
$$

is called fractional-order accumulative linear timevarying parameters discrete grey model (FTDGM).

Theorem 7. The parameters of the FTDGM(1,1) model can be solved by using the following least-squares estimation:

$$
\left[\begin{array}{l}
\beta_{1} \\
\beta_{2} \\
\beta_{3} \\
\beta_{4}
\end{array}\right]=\left(D^{T} D\right)^{-1} D^{T} W
$$

and, among them,

$$
\begin{aligned}
D & =\left[\begin{array}{cccc}
x^{(r)}(1) & x^{(r)}(1) & 1 & 1 \\
2 x^{(r)}(2) & x^{(r)}(2) & 2 & 1 \\
\vdots & \vdots & \vdots & \vdots \\
(n-2) x^{(r)}(n-2) & x^{(r)}(n-2) & n-2 & 1 \\
(n-1) x^{(r)}(n-1) & x^{(r)}(n-1) & n-1 & 1
\end{array}\right], \\
W & {\left[\begin{array}{c}
x^{(r)}(2) \\
x^{(r)}(3) \\
\vdots \\
x^{(r)}(n-1) \\
x^{(r)}(n)
\end{array}\right] . }
\end{aligned}
$$

The predicted value of FTDGM(1,1)model is as follows:

$$
\widehat{x}^{(r)}(k+1)=\left(\beta_{1} k+\beta_{2}\right) \hat{x}^{(r)}(k)+\beta_{3} k+\beta_{4}
$$

According to the calculation formula of fractional-order accumulation, it is not difficult to calculate the reduced value of the predicted sequence as follows:

$$
\widehat{x}^{(0)}(k)=\widehat{x}^{(r)}(k)-\sum_{i=1}^{k} C_{k-i+r-1}^{k-i} \widehat{x}^{(r)}(i),
$$

$$
k=1,2, \ldots, n, \ldots
$$

\section{Disturbance Analysis of TDGM(1,1) Model and FTDGM(1,1) Model}

Theorem 8 (see $[36,37]$ ). Suppose that $A \in C^{m \times n}, b \in C^{m}, A^{\dagger}$ is the generalized inverse matrix of $A$. When the column vector of $A$ has linear independence, the function $\|A x-b\|_{2}=\min$ has a unique solution.
Theorem 9 (see $[36,37])$. Suppose that $A \in C^{m \times n}, b \in C^{m}, A^{\dagger}$ is the generalized inverse matrix of $A . B=A+E, c=b+k \epsilon$ $C^{n}$. Suppose that the solutions of function $\|B x-c\|_{2}=\min$ and $\|A x-b\|_{2}=\min$ are $x+h$ and $x$, respectively. When $\operatorname{rank}(A)=\operatorname{rank}(B)=n$ and $\left\|A^{\dagger}\right\|_{2}\|E\|_{2}<1$, we have the following result:

$$
\|h\|_{2} \leq \frac{s_{\dagger}}{t_{\dagger}}\left(\frac{\|E\|_{2}}{\|A\|}\|x\|+\frac{\|k\|}{\|A\|}+\frac{s_{\dagger}}{t_{\dagger}} \frac{\|E\|_{2}}{\|A\|} \frac{\left\|r_{x}\right\|}{\|A\|}\right) .
$$

Among them,

$$
\begin{aligned}
& s_{\dagger}=\left\|A^{\dagger}\right\|_{2}\|A\|, \\
& t_{\dagger}=1-\left\|A^{\dagger}\right\|_{2}\|E\|_{2}, \\
& r_{x}=b-A x .
\end{aligned}
$$

3.1. Disturbance Analysis of TDGM(1,1) Model. The perturbation bounds of $\operatorname{TDGM}(1,1)$ model will be analyzed in the following section.

Theorem 10. The solution of TDGM model can be given as the following function: $\|Y-C x\|_{2}=\min$. Suppose that the solution of the TDGM $(1,1)$ model is $x$, and $\widehat{x}^{(0)}(1)=x^{(0)}(1)+\varepsilon$. Among them, $\varepsilon$ is the disturbance information.

Then

$$
\begin{gathered}
\|h\|_{2} \leq|\varepsilon| \frac{s_{\dagger}}{t_{\dagger}}\left(\frac{\|x\| \sqrt{\sum_{i=1}^{n-1} i^{2}}}{\|B\|}+\frac{\sqrt{n-1}}{\|B\|}\right. \\
\left.+\frac{s_{\dagger}}{t_{\dagger}} \frac{\sqrt{\sum_{i=1}^{n-1} i^{2}}}{\|B\|} \frac{\left\|r_{x}\right\|}{\|B\|}\right) .
\end{gathered}
$$

Proof.

$\widehat{C}=C+\Delta C$

$=\left[\begin{array}{cccc}x^{(1)}(1)+\varepsilon & x^{(1)}(1)+\varepsilon & 1 & 1 \\ 2\left(x^{(1)}(2)+\varepsilon\right) & x^{(1)}(2)+\varepsilon & 2 & 1 \\ \vdots & \vdots & \vdots & \vdots \\ (n-2)\left(x^{(1)}(n-2)+\varepsilon\right) & x^{(1)}(n-2)+\varepsilon & n-2 & 1 \\ (n-1)\left(x^{(1)}(n-1)+\varepsilon\right) & x^{(1)}(n-1)+\varepsilon & n-1 & 1\end{array}\right]$

$$
=\left[\begin{array}{cccc}
x^{(1)}(1) & x^{(1)}(1) & 1 & 1 \\
2 x^{(1)}(2) & x^{(1)}(2) & 2 & 1 \\
\vdots & \vdots & \vdots & \vdots \\
(n-2) x^{(1)}(n-2) & x^{(1)}(n-2) & n-2 & 1 \\
(n-1) x^{(1)}(n-1) & x^{(1)}(n-1) & n-1 & 1
\end{array}\right]
$$




$$
\begin{gathered}
+\left[\begin{array}{cccc}
\varepsilon & \varepsilon & 0 & 0 \\
2 \varepsilon & \varepsilon & 0 & 0 \\
\vdots & \vdots & \vdots & \vdots \\
(n-2) \varepsilon & \varepsilon & 0 & 0 \\
(n-1) \varepsilon & \varepsilon & 0 & 0
\end{array}\right] \\
\widehat{Y}=Y+\Delta Y=\left[\begin{array}{c}
x^{(1)}(2) \\
x^{(1)}(3) \\
\vdots \\
x^{(1)}(n-1) \\
x^{(1)}(n)
\end{array}\right]+\left[\begin{array}{c}
\varepsilon \\
\varepsilon \\
\vdots \\
\varepsilon \\
\varepsilon
\end{array}\right] .
\end{gathered}
$$

Assume that the solution of the new model $\|\widehat{Y}-\widehat{C} x\|_{2}=$ $\min$ is $\widehat{x}$, and the disturbance is $h$. The equation $\|Y-C x\|_{2}=$ min has a unique solution $x=Y^{\dagger} C$ due to the linear independence of column vectors of $C$.

Since

$$
\begin{aligned}
\Delta Y & =\left[\begin{array}{c}
\varepsilon \\
\varepsilon \\
\vdots \\
\varepsilon \\
\varepsilon
\end{array}\right], \\
\Delta C^{T} \Delta C & =\left[\begin{array}{llll}
\sum_{i=1}^{n-1} i^{2} \varepsilon^{2} & \sum_{i=1}^{n-1} i \varepsilon^{2} & 0 & 0 \\
\sum_{i=1}^{n-1} & (n-1) \varepsilon^{2} & 0 & 0 \\
0 & 0 & 0 & 0 \\
0 & 0 & 0 & 0
\end{array}\right] . \\
\|\Delta Y\|_{2} & =|\varepsilon| \sqrt{n-1}, \\
\|\Delta C\|_{2} & =\sqrt{\lambda_{\max }\left(\Delta C^{T} \Delta C\right),}
\end{aligned}
$$

we have

$$
\|\Delta C\|_{2}=\sqrt{\sum_{i=1}^{n-1} i^{2} \varepsilon^{2}}=|\varepsilon| \sqrt{\sum_{i=1}^{n-1} i^{2}}
$$

Then, the following result can be obtained according to Theorem 9:

$$
\begin{gathered}
\|h\|_{2} \leq \frac{s_{\dagger}}{t_{\dagger}}\left(\frac{\|\Delta C\|_{2}}{\|B\|}\|x\|+\frac{\|\Delta Y\|}{\|B\|}+\frac{s_{\dagger}}{t_{\dagger}} \frac{\|\Delta C\|_{2}}{\|B\|} \frac{\left\|r_{x}\right\|}{\|B\|}\right) \\
=|\varepsilon| \frac{s_{\dagger}}{t_{\dagger}}\left(\frac{\|x\| \sqrt{\sum_{i=1}^{n-1} i^{2}}}{\|B\|}+\frac{\sqrt{n-1}}{\|B\|}\right. \\
\left.+\frac{s_{\dagger}}{t_{\dagger}} \frac{\sqrt{\sum_{i=1}^{n-1} i^{2}}}{\|B\|} \frac{\left\|r_{x}\right\|}{\|B\|}\right)=Q\left(x^{(0)}(1)\right) .
\end{gathered}
$$

Theorem 11. Assume that the conditions of Theorem 9 remain unchanged, and $\widehat{x}^{(0)}(t)=x^{(0)}(t)+\varepsilon$.

Then the perturbation bound of the solution is as follows:

$$
\begin{gathered}
\|h\|_{2} \leq|\varepsilon| \frac{s_{\dagger}}{t_{\dagger}}\left(\frac{\|x\| \sqrt{\sum_{i=t}^{n-1} i^{2}}}{\|B\|}+\frac{\sqrt{n-t+1}}{\|B\|}\right. \\
\left.+\frac{s_{\dagger}}{t_{\dagger}} \frac{\sqrt{\sum_{i=t}^{n-1} i^{2}}}{\|B\|} \frac{\left\|r_{x}\right\|}{\|B\|}\right) .
\end{gathered}
$$

Proof.

$\widehat{C}=C+\Delta C$

$=\left[\begin{array}{cccc}x^{(1)}(1) & x^{(1)}(1) & 1 & 1 \\ \vdots & \vdots & \vdots & \vdots \\ t\left(x^{(1)}(t)+\varepsilon\right) & x^{(1)}(t)+\varepsilon & t & 1 \\ \vdots & \vdots & \vdots & \vdots \\ (n-2)\left(x^{(1)}(n-2)+\varepsilon\right) & x^{(1)}(n-2)+\varepsilon & n-2 & 1 \\ (n-1)\left(x^{(1)}(n-1)+\varepsilon\right) & x^{(1)}(n-1)+\varepsilon & n-1 & 1\end{array}\right]$

$=\left[\begin{array}{cccc}x^{(1)}(1) & x^{(1)}(1) & 1 & 1 \\ \vdots & \vdots & \vdots & \vdots \\ t\left(x^{(1)}(t)\right. & x^{(1)}(t) & t & 1 \\ \vdots & \vdots & \vdots & \vdots \\ (n-2)\left(x^{(1)}(n-2)\right) & x^{(1)}(n-2) & n-2 & 1 \\ (n-1)\left(x^{(1)}(n-1)\right) & x^{(1)}(n-1) & n-1 & 1\end{array}\right]$

$+\left[\begin{array}{cccc}0 & 0 & 0 & 0 \\ \vdots & \vdots & \vdots & \vdots \\ t \varepsilon & \varepsilon & 0 & 0 \\ \vdots & \vdots & \vdots & \vdots \\ (n-2) \varepsilon & \varepsilon & 0 & 0 \\ (n-1) \varepsilon & \varepsilon & 0 & 0\end{array}\right]$

$$
\widehat{Y}=Y+\Delta Y=\left[\begin{array}{c}
x^{(1)}(2) \\
\vdots \\
x^{(1)}(t) \\
\vdots \\
x^{(1)}(n-1) \\
x^{(1)}(n)
\end{array}\right]+\left[\begin{array}{c}
0 \\
\vdots \\
\varepsilon \\
\vdots \\
\varepsilon
\end{array}\right]
$$

Assume that the solution of the new model $\| \widehat{Y}-$ $\widehat{C} x \|_{2}=\min$ is $\widehat{x}$, and the disturbance is $h$. The equation 
$\|Y-C x\|_{2}=\min$ has a unique solution $x=Y^{\dagger} C$ due to the linear independence of column vectors of $C$.

Since

$$
\begin{aligned}
& \Delta Y=\left[\begin{array}{c}
0 \\
\vdots \\
\varepsilon \\
\vdots \\
\varepsilon \\
\varepsilon
\end{array}\right], \\
& \Delta C^{T} \Delta C=\left[\begin{array}{cccc}
\sum_{i=t}^{n-1} i^{2} \varepsilon^{2} & \sum_{i=t}^{n-1} i \varepsilon^{2} & 0 & 0 \\
\sum_{i=t}^{n-1} i \varepsilon^{2} & (n-t+1) \varepsilon^{2} & 0 & 0 \\
0 & 0 & 0 & 0 \\
0 & 0 & 0 & 0
\end{array}\right] \text {. } \\
& \|\Delta Y\|_{2}=|\varepsilon| \sqrt{n-t+1}, \\
& \|\Delta C\|_{2}=\sqrt{\lambda_{\max }\left(\Delta C^{T} \Delta C\right)},
\end{aligned}
$$

we have

$$
\|\Delta C\|_{2}=\sqrt{\sum_{i=t}^{n-1} i^{2} \varepsilon^{2}}=|\varepsilon| \sqrt{\sum_{i=t}^{n-1} i^{2}}
$$

Then, the following result can be obtained according to Theorem 9:

$$
\begin{gathered}
\|h\|_{2} \leq \frac{s_{\dagger}}{t_{\dagger}}\left(\frac{\|\Delta C\|_{2}}{\|B\|}\|x\|+\frac{\|\Delta Y\|}{\|B\|}+\frac{s_{\dagger}}{t_{\dagger}} \frac{\|\Delta C\|_{2}}{\|B\|} \frac{\left\|r_{x}\right\|}{\|B\|}\right) \\
=|\varepsilon| \frac{s_{\dagger}}{t_{\dagger}}\left(\frac{\|x\| \sqrt{\sum_{i=t}^{n-1} i^{2}}}{\|B\|}+\frac{\sqrt{n-t+1}}{\|B\|}\right. \\
\left.+\frac{s_{\dagger}}{t_{\dagger}} \frac{\sqrt{\sum_{i=t}^{n-1} i^{2}}}{\|B\|} \frac{\left\|r_{x}\right\|}{\|B\|}\right)=Q\left(x^{(1)}(t)\right) .
\end{gathered}
$$

3.2. Disturbance Analysis of $\operatorname{TDGM}(1,1)$ Model and FTDGM(1,1) Model

Theorem 12. The solution of $\operatorname{TDGM}(1,1)$ model can be given as the following function: $\|W-D x\|_{2}=\min$. Suppose that the solution of the TDGM $(1,1)$ model is $x$, and $\widehat{x}^{(0)}(1)=x^{(0)}(1)+\varepsilon$. Among them, $\varepsilon$ is the disturbance information.
Then

$$
\begin{aligned}
\|h\|_{2} \leq|\varepsilon| \frac{s_{\dagger}}{t_{\dagger}}\left(\frac{\sqrt{\sum_{i=1}^{n-1}\left(i C_{i+r-2}^{i-1}\right)^{2}}}{\|B\|}\|x\|\right. \\
\left.+\frac{\sqrt{\sum_{i=1}^{n-1}\left(C_{i+r-2}^{i-1}\right)^{2}}}{\|B\|}+\frac{s_{\dagger}}{t_{\dagger}} \frac{\sqrt{\sum_{i=1}^{n-1}\left(i C_{i+r-2}^{i-1}\right)^{2}}}{\|B\|} \frac{\left\|r_{x}\right\|}{\|B\|}\right) .
\end{aligned}
$$

Proof.

$$
\widehat{D}=D+\Delta D
$$$$
=\left[\begin{array}{cccc}
x^{(r)}(1) & x^{(r)}(1) & 1 & 1 \\
2 x^{(r)}(2) & x^{(r)}(2) & 2 & 1 \\
\vdots & \vdots & \vdots & \vdots \\
(n-2) x^{(r)}(n-2) & x^{(r)}(n-2) & n-2 & 1 \\
(n-1) x^{(r)}(n-1) & x^{(r)}(n-1) & n-1 & 1
\end{array}\right]
$$

$$
+\left[\begin{array}{cccc}
\varepsilon & \varepsilon & 0 & 0 \\
2 r \varepsilon & r \varepsilon & 0 & 0 \\
\vdots & \vdots & \vdots & \vdots \\
(n-2) C_{n-4+r}^{n-3} \varepsilon & C_{n-4+r}^{n-3} \varepsilon & 0 & 0 \\
(n-1) C_{n-3+r}^{n-2} \varepsilon & C_{n-3+r}^{n-2} \varepsilon & 0 & 0
\end{array}\right]
$$

$$
\widehat{W}=W+\Delta W=\left[\begin{array}{c}
x^{(r)}(2) \\
x^{(r)}(3) \\
\vdots \\
x^{(r)}(n-1) \\
x^{(r)}(n)
\end{array}\right]+\left[\begin{array}{c}
r \varepsilon \\
C_{1+r}^{2} \varepsilon \\
\vdots \\
C_{n-3+r}^{n-2} \varepsilon \\
C_{n-2+r}^{n-1} \varepsilon
\end{array}\right] .
$$

Assume that the solution of the new model $\|\widehat{Y}-\widehat{B} x\|_{2}=$ $\min$ is $\widehat{x}$, and the disturbance is $h$. The equation $\|Y-B x\|_{2}=$ min has a unique solution $x=Y^{\dagger} B$ due to the linear independence of column vectors of $B$.

Since

$$
\Delta W=\left[\begin{array}{c}
r \varepsilon \\
C_{1+r}^{2} \varepsilon \\
\vdots \\
C_{n-3+r}^{n-2} \varepsilon \\
C_{n-2+r}^{n-1} \varepsilon
\end{array}\right]
$$




$$
\Delta D^{T} \Delta D=\left[\begin{array}{cccc}
\sum_{i=1}^{n-1}\left(i C_{i+r-2}^{i-1} \varepsilon\right)^{2} & \sum_{i=1}^{n-1} i\left(C_{i+r-2}^{i-1} \varepsilon\right)^{2} & 0 & 0 \\
\sum_{i=1}^{n-1} i\left(C_{i+r-2}^{i-1} \varepsilon\right)^{2} & \sum_{i=1}^{n-1}\left(C_{i+r-2}^{i-1} \varepsilon\right)^{2} & 0 & 0 \\
0 & 0 & 0 & 0 \\
0 & 0 & 0 & 0
\end{array}\right]
$$$$
\|\Delta W\|_{2}=|\varepsilon| \sqrt{\sum_{i=2}^{n}\left(C_{i+r-2}^{i-1}\right)^{2}},
$$$$
\|\Delta D\|_{2}=\sqrt{\lambda_{\max }\left(\Delta D^{T} \Delta D\right)}
$$

we have

$$
\begin{aligned}
& \|\Delta W\|_{2}=|\varepsilon| \sqrt{\sum_{i=2}^{n}\left(C_{i+r-2}^{i-1}\right)^{2}}, \\
& \|\Delta D\|_{2}=\sqrt{\sum_{i=1}^{n-1}\left(i C_{i+r-2}^{i-1} \varepsilon\right)^{2}}=|\varepsilon| \sqrt{\sum_{i=1}^{n-1} i^{2}\left(C_{i+r-2}^{i-1}\right)^{2}}
\end{aligned}
$$

Then, the following result can be obtained according to Theorem 9:

$$
\begin{aligned}
& \|h\|_{2} \leq \frac{s_{\dagger}}{t_{\dagger}}\left(\frac{\|\Delta D\|_{2}}{\|B\|}\|x\|+\frac{\|\Delta Y\|}{\|B\|}+\frac{s_{\dagger}}{t_{\dagger}} \frac{\|\Delta D\|_{2}}{\|D\|} \frac{\left\|r_{x}\right\|}{\|B\|}\right) \\
& =|\varepsilon| \frac{s_{\dagger}}{t_{\dagger}}\left(\frac{\sqrt{\sum_{i=1}^{n-1}\left(i C_{i+r-2}^{i-1}\right)^{2}}}{\|B\|}\|x\|+\frac{\sqrt{\sum_{i=2}^{n}\left(i_{i+r-2}^{i-1}\right)^{2}}}{\|B\|}\right. \\
& \left.+\frac{s_{\dagger}}{t_{\dagger}} \frac{\left.\sqrt{\sum_{i=1}^{n-1}\left(i C_{i+r-2}^{i-1}\right)^{2}} \frac{\left\|r_{x}\right\|}{\|B\|}\right)=L\left(x^{(0)}(1)\right)}{\|B\|}\right) \\
& Q\left(x^{(0)}(1)\right)=|\varepsilon| \frac{s_{\dagger}}{t_{\dagger}}\left(\frac{\|x\| \sqrt{\sum_{i=1}^{n-1} i^{2}}}{\|B\|}+\frac{\sqrt{n-1}}{\|B\|}\right. \\
& \left.+\frac{s_{\dagger}}{t_{\dagger}} \frac{\sqrt{\sum_{i=1}^{n-1} i^{2}}}{\|B\|} \frac{\left\|r_{x}\right\|}{\|B\|}\right)
\end{aligned}
$$

Since

$$
C_{i+r-2}^{i-1}=C_{i-1+r-1}^{i-1}<1
$$

we have

$$
\begin{aligned}
\sqrt{\sum_{i=1}^{n-1} i^{2}\left(C_{i+r-2}^{i-1} \varepsilon\right)^{2}} & <\sqrt{\sum_{i=1}^{n-1} i^{2}} \\
\sqrt{\sum_{i=2}^{n}\left(C_{i+r-2}^{i-1}\right)^{2}} & <\sqrt{n-1} .
\end{aligned}
$$

It is not hard to get $L\left(x^{(0)}(1)\right)<Q\left(x^{(0)}(1)\right)$.

Theorem 13. The solution of $\operatorname{TDGM}(1,1)$ model can be given as the following function: $\|W-D x\|_{2}=\min$. Suppose that the solution of the TDGM $(1,1)$ model is $x$, and $\hat{x}^{(0)}(t)=x^{(0)}(t)+\varepsilon$. Among them, $\varepsilon$ is the disturbance information. Then

$$
\begin{gathered}
\|h\|_{2} \leq|\varepsilon| \frac{s_{\dagger}}{t_{\dagger}}\left(\frac{\sqrt{\sum_{i=\mathrm{t}}^{n-1}\left(i C_{i+r-t-1}^{i-t}\right)^{2}}}{\|B\|}\|x\|\right. \\
+\frac{\sqrt{\sum_{i=t}^{n}\left(C_{i-t+r-1}^{i-t}\right)^{2}}}{\|B\|}
\end{gathered}
$$

$$
\left.+\frac{s_{\dagger}}{t_{\dagger}} \frac{\sqrt{\sum_{i=t}^{n-1}\left(i C_{i+r-t-1}^{i-t}\right)^{2}}}{\|B\|} \frac{\left\|r_{x}\right\|}{\|B\|}\right)=L\left(x^{(0)}(t)\right) .
$$

Proof.

$$
\widehat{D}=D+\Delta D
$$

$$
=\left[\begin{array}{cccc}
x^{(r)}(1) & x^{(r)}(1) & 1 & 1 \\
\vdots & \vdots & \vdots & \vdots \\
t\left(x^{(r)}(t)\right. & x^{(r)}(t) & t & 1 \\
\vdots & \vdots & \vdots & \vdots \\
(n-2)\left(x^{(r)}(n-2)\right) & x^{(r)}(n-2) & n-2 & 1 \\
(n-1)\left(x^{(r)}(n-1)\right) & x^{(r)}(n-1) & n-1 & 1
\end{array}\right]
$$

$$
+\left[\begin{array}{cccc}
0 & 0 & 1 & 1 \\
\vdots & \vdots & \vdots & \vdots \\
t \varepsilon & \varepsilon & 0 & 0 \\
\vdots & \vdots & \vdots & \vdots \\
(n-2) C_{n-t+r-3}^{n-t-2} \varepsilon & C_{n-t+r-3}^{n-t-2} \varepsilon & 0 & 0 \\
(n-1) C_{n-t+r-2}^{n-t-1} \varepsilon & C_{n-t+r-2}^{n-t-1} \varepsilon & 0 & 0
\end{array}\right]
$$

$$
\widehat{W}=W+\Delta W=\left[\begin{array}{c}
x^{(r)}(2) \\
\vdots \\
x^{(r)}(t) \\
\vdots \\
x^{(r)}(n-1) \\
x^{(r)}(n)
\end{array}\right]+\left[\begin{array}{c}
0 \\
\vdots \\
\varepsilon \\
\vdots \\
C_{n-t+r-3}^{n-t-2} \\
C_{n-t+r-2}^{n-t-1} \varepsilon
\end{array}\right]
$$


We have

$$
\Delta W=\left[\begin{array}{c}
0 \\
\vdots \\
\varepsilon \\
\vdots \\
C_{n-t+r-3}^{n-t-2} \varepsilon \\
C_{n-t+r-2}^{n-t-1} \varepsilon
\end{array}\right],
$$$$
\Delta D^{T} \Delta D
$$

$$
=\left[\begin{array}{cccc}
\sum_{i=t}^{n-1}\left(i C_{i-t+r-1}^{i-t} \varepsilon\right)^{2} & \sum_{i=t}^{n-1} i\left(C_{i-t+r-1}^{i-t} \varepsilon\right)^{2} & 0 & 0 \\
\sum_{i=t}^{n-1} i\left(C_{i-t+r-1}^{i-t} \varepsilon\right)^{2} & \sum_{i=t}^{n-1}\left(C_{i-t+r-1}^{i-t} \varepsilon\right)^{2} & 0 & 0 \\
0 & 0 & 0 & 0 \\
0 & 0 & 0 & 0
\end{array}\right] .
$$

$$
\begin{aligned}
& \|\Delta W\|_{2}=|\varepsilon| \sqrt{\sum_{i=t}^{n}\left(C_{i-t+r-1}^{i-t}\right)^{2}}, \\
& \|\Delta D\|_{2}=\sqrt{\lambda_{\max }\left(\Delta D^{T} \Delta D\right)}=|\varepsilon| \sqrt{\sum_{i=\mathrm{t}}^{n-1}\left(i C_{i-t+r-1}^{i-t}\right)^{2}}
\end{aligned}
$$

Then, the following result can be obtained according to Theorem 9.

$$
\begin{gathered}
\|h\|_{2} \leq \frac{s_{\dagger}}{t_{\dagger}}\left(\frac{\|\Delta D\|_{2}}{\|B\|}\|x\|+\frac{\|\Delta W\|}{\|B\|}+\frac{s_{\dagger}}{t_{\dagger}} \frac{\|\Delta D\|_{2}}{\|D\|} \frac{\left\|r_{x}\right\|}{\|B\|}\right) \\
=|\varepsilon| \frac{s_{\dagger}}{t_{\dagger}}\left(\frac{\sqrt{\sum_{i=t}^{n-1}\left(i C_{i-t+r-1}^{i-t}\right)^{2}}}{\|B\|}\|x\|\right. \\
+\frac{\sqrt{\sum_{i=t}^{n}\left(C_{i-t+r-1}^{i-t}\right)^{2}}}{\|B\|} \\
\left.+\frac{s_{\dagger}}{t_{\dagger}} \frac{\sqrt{\sum_{i=t}^{n-1}\left(i C_{i-t+r-1}^{i-t}\right)^{2}}}{\|B\|} \frac{\left\|r_{x}\right\|}{\|B\|}\right)=L\left(x^{(0)}(t)\right)
\end{gathered}
$$

We have

$$
\begin{aligned}
& Q\left(x^{(0)}(t)\right)=|\varepsilon| \frac{s_{\dagger}}{t_{\dagger}}\left(\frac{\|x\| \sqrt{\sum_{i=t}^{n-1} i^{2}}}{\|B\|}+\frac{\sqrt{n-t+1}}{\|B\|}\right. \\
& \left.\quad+\frac{s_{\dagger}}{t_{\dagger}} \frac{\sqrt{\sum_{i=t}^{n-1} i^{2}}}{\|B\|} \frac{\left\|r_{x}\right\|}{\|B\|}\right)
\end{aligned}
$$

$$
\begin{aligned}
& \sum_{i=1}^{n-t}\left(i C_{k+r-2}^{i-1}\right)^{2}<\sum_{i=1}^{n-t} i^{2}<\sum_{i=t}^{n-1} i^{2} \\
& \sum_{i=1}^{n-t+1}\left(C_{k+r-2}^{i-1}\right)^{2}<n-t+1 .
\end{aligned}
$$

Then $L\left(x^{(0)}(t)\right)<Q\left(x^{(0)}(t)\right)$.

It can be seen from the conclusion of Theorems 12 and 13 that when $r<1$, the disturbance bound of FTDGM $(1,1)$ model is smaller than that of TDGM $(1,1)$ model. Generally speaking, compared with the TDGM(1,1) model, the FTDGM $(1,1)$ model has better robustness. The proposed FTDGM(1,1) model can effectively reduce the prediction error caused by system disturbance and improve the prediction accuracy of the grey forecasting model. And the solution of optimal parameter $r$ can be given by genetic algorithms.

\section{Numerical Illustrations}

In order to test the modeling effect of the model, two real cases will be given in the following section.

Case 1. The GDP of Guangdong province in 2001-2009 is used to build different grey prediction model. The data is shown in Table 1. Different grey prediction models were established based on the given data. And the advantages of the model were tested by comparing the prediction accuracies of different models.

The highest prediction accuracy in [38] is time-varying parameters grey model (TVGM(1,1) model). This Paper established $\operatorname{DGM}(1,1)$ model, $\operatorname{TVGM}(1,1)$ model, and FTDGM(1,1) model, respectively. The calculation results are shown in Table 2 and Figure 1. The parameters of $\operatorname{FTDGM}(1,1)$ model are as follows: $\beta_{1}=0.0185, \beta_{2}=$ $0.5166, \beta_{3}=3530.48, \beta_{4}=7817.70, r=0.41$. It can be seen from Table 2 that, because of the nature of exponential function, large errors will appear after the second prediction of the DGM(1,1) model. TVGM(1,1) model can effectively increase the prediction accuracy along with adjusting and optimizing the time-varying parameters. However, the prediction error is still relatively large. The proposed FTDGM(1,1) model in this paper shows good robustness, and the prediction error does not increase with the passage of time. It indicated that the $\operatorname{FTDGM}(1,1)$ model has better anti-interference and long-term memory, which can be used to predict medium-term goals.

Case 2. As one of the important symbols of China's transportation modernization, highway reflects the degree and level of a country's modernization. Compared with railway and air or water transportation, highway transportation is the more used mode in passenger and cargo transportation. Highway transportation is point-to-point direct, flexible, and convenient. It is very important to accurately predict the length of highway transportation route. The length of Chinese highway transportation route in 2010-2017 is used to build 


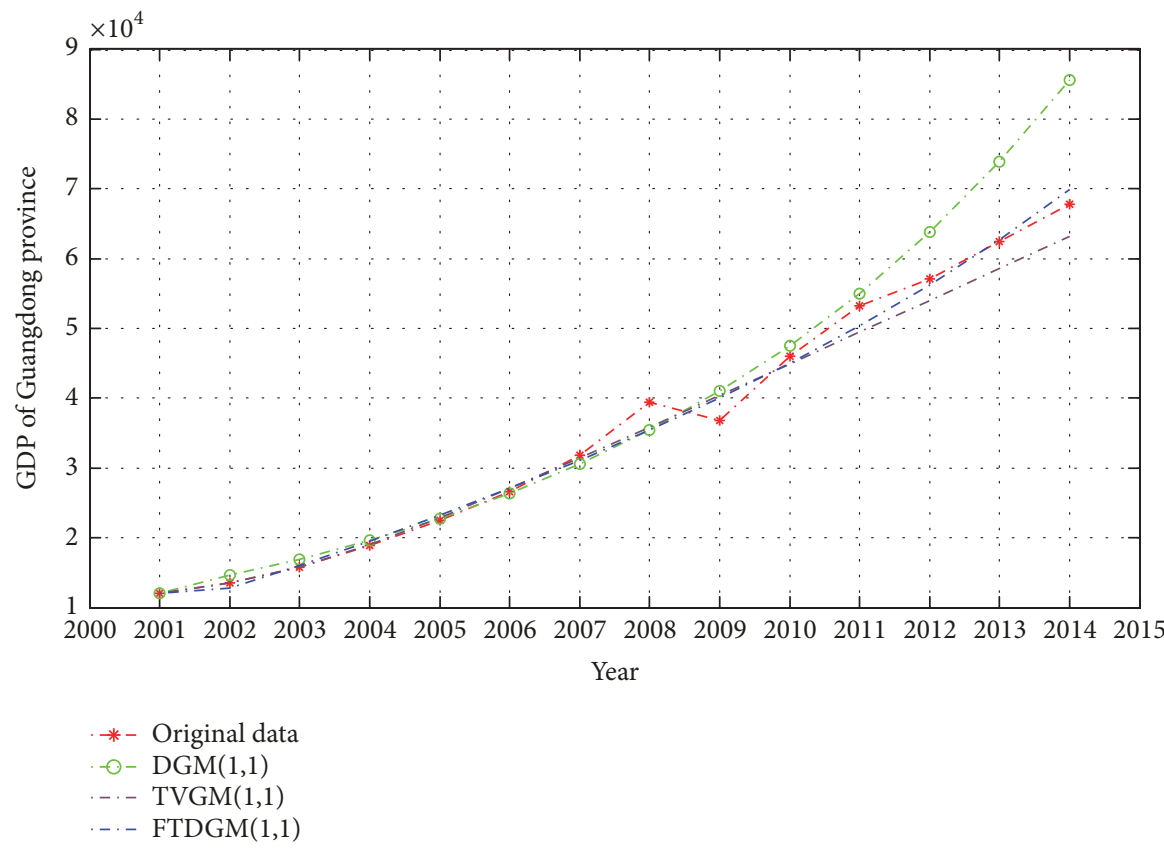

FIGURE 1: Calculation results of different models of Case 1.

TABLE 1: Gross domestic product in Guangdong province in 2001-2014 (one hundred million yuan).

\begin{tabular}{lccccccc}
\hline Year & 2001 & 2002 & 2003 & 2004 & 2005 & 2006 & 2007 \\
\hline GDP & 12039.25 & 13502.42 & 15844.64 & 18864.62 & 22557.37 & 26587.76 & 31777.01 \\
\hline Year & 2008 & 2009 & 2010 & 2011 & 2012 & 2013 & 2014 \\
\hline GDP & 39482.56 & 36796.71 & 46013 & 53210 & 57068 & 62475 & 67792 \\
\hline
\end{tabular}

Source: Guangdong Statistical Yearbook.

TABLE 2: Predicted values and predicted errors of different grey prediction models.

\begin{tabular}{lccccccc}
\hline \multirow{2}{*}{ Year } & \multirow{2}{*}{ Original value } & \multicolumn{2}{c}{ DGM(1,1) model } & \multicolumn{2}{c}{ TVGM(1,1) model } & \multicolumn{2}{c}{ FTDGM(1,1) model } \\
& & Predicted value & Relative error (\%) & Predicted value & Relative error (\%) & Predicted value & Relative error (\%) \\
\hline 2010 & 46013 & 48326.89 & 5.03 & 44920.35 & 2.37 & 44989.65 & 2.22 \\
2011 & 53210 & 56295.98 & 5.80 & 49471.34 & 7.03 & 50379.20 & 5.32 \\
2012 & 57068 & 65579.18 & 14.91 & 54030.11 & 5.32 & 56267.53 & 1.40 \\
2013 & 62475 & 76393.18 & 22.28 & 58593.05 & 6.21 & 62739.40 & 0.42 \\
2014 & 67792 & 88990.4 & 31.27 & 63158.22 & 6.83 & 69893.15 & 3.10 \\
MAPE & & & 15.86 & & 5.55 & 2.49 \\
\hline
\end{tabular}

MAPE (mean absolute percentage error) $=100 \%(1 / n) \sum_{k=1}^{n}|(x(k)-\widehat{x}(k)) / x(k)|$.

different grey prediction model. The unit of highway mileage is ten thousand kilometers. The calculation results of different models are shown in Table 3 and Figure 2. The parameters of $\operatorname{FTDGM}(1,1)$ model are as follows: $\beta_{1}=0.06, \beta_{2}=$ $-1.46, \beta_{3}=17.44, \beta_{4}=7.76, r=0.85$.

It can be seen from the calculation results that the $\operatorname{TDGM}(1,1)$ model is better than the $\operatorname{DGM}(1,1)$ model in describing the internal evolution. However, neither the $\operatorname{DGM}(1,1)$ model nor the $\operatorname{TDGM}(1,1)$ model can accurately describe the development trend of the system. In particular, the prediction error of the second step is relatively large, indicating that the memory of the integer-order model is insufficient for the FTDGM $(1,1)$ model. The prediction error of the FTDGM(1,1) model is only $0.64 \%$, which shows that the model has strong extrapolation ability and good memory.

\section{Concluding Remarks}

On the basis of the traditional discrete grey prediction model, this paper proposed the FTDGM $(1,1)$ model. The parametric solution method of the model was given. By using the matrix disturbance theory, the disturbance boundary of the model 
TABLE 3: The calculation results of different grey models.

\begin{tabular}{|c|c|c|c|c|c|c|c|}
\hline \multirow{2}{*}{ Year } & \multirow{2}{*}{ Original value } & \multicolumn{2}{|c|}{$\operatorname{DGM}(1,1)$ model } & \multicolumn{2}{|c|}{$\operatorname{TDGM}(1,1)$ model } & \multicolumn{2}{|c|}{ FTDGM $(1,1)$ model, $r=0.85$} \\
\hline & & Simulation value & Relative error(\%) & Simulation value & Relative error (\%) & Simulation value & Relative error (\%) \\
\hline 2010 & 7.41 & 7.41 & 0 & 7.41 & 0 & 7.41 & 0 \\
\hline 2011 & 8.49 & 8.65 & 1.91 & 8.49 & 0.05 & 8.49 & 0.00 \\
\hline 2012 & 9.62 & 9.46 & 1.69 & 9.65 & 0.30 & 9.64 & 0.23 \\
\hline 2013 & 10.44 & 10.34 & 0.98 & 10.37 & 0.71 & 10.33 & 1.07 \\
\hline 2014 & 11.19 & 11.30 & 0.97 & 11.28 & 0.80 & 11.45 & 2.29 \\
\hline 2015 & 12.35 & 12.35 & 0.00 & 12.30 & 0.42 & 11.97 & 3.06 \\
\hline MAPE & & & 1.11 & & 0.46 & & 1.33 \\
\hline 2016 & 13.1 & 13.50 & 3.05 & 13.46 & 2.73 & 13.22 & 0.92 \\
\hline 2017 & 13.64 & 14.76 & 8.18 & 14.78 & 8.36 & 13.69 & 0.37 \\
\hline MAPE & & & 5.61 & & 5.54 & & 0.64 \\
\hline
\end{tabular}

Source: China Statistical Yearbook.

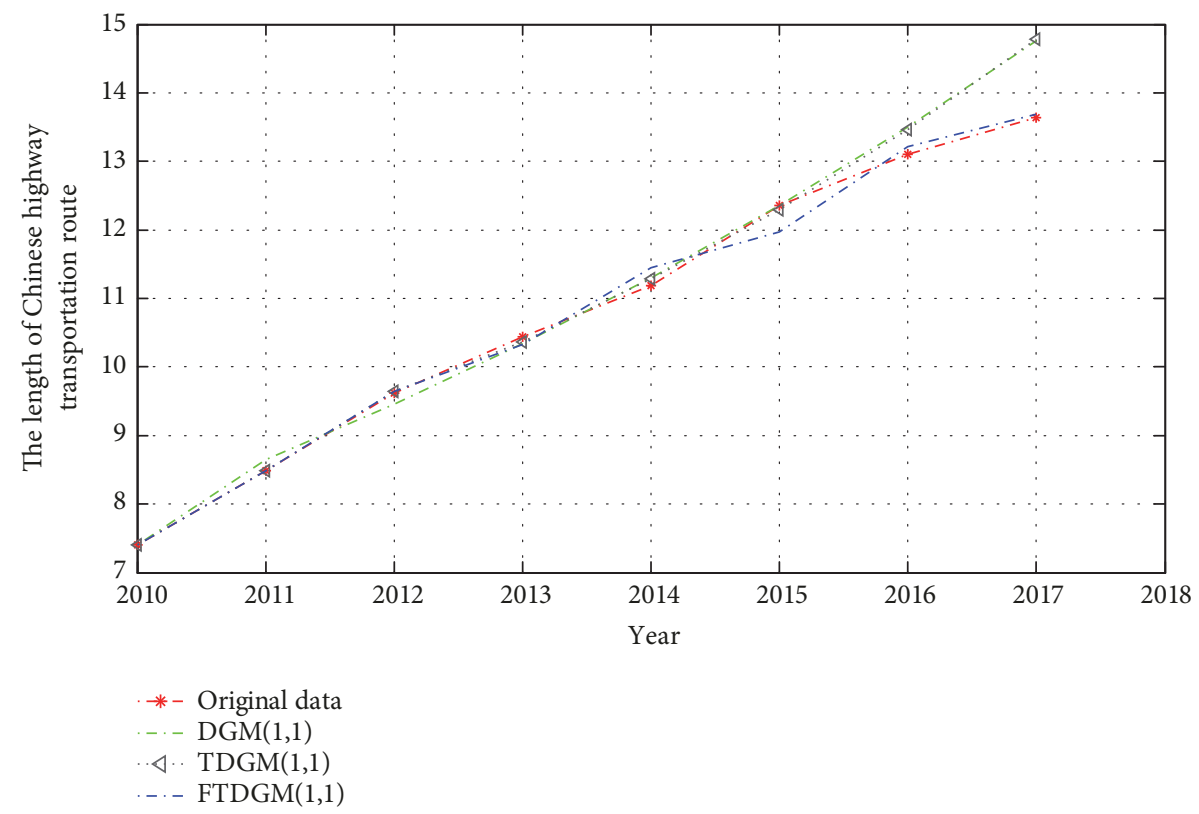

FIGURE 2: Calculation results of different models of Case 2.

was analyzed, and it was proved that the FTDGM(1,1) model had better robustness than the TDGM $(1,1)$ model.

Two real cases were used to test the effect of the proposed FTDGM(1,1) model. It was found that the prediction accuracy is higher than that of the existing models, which further verifies the superiority of the proposed FTDGM(1,1) model. At the same time, the FTDGM $(1,1)$ model had better memory than other discrete grey prediction models. Research results of this paper further expand the application scope of the grey prediction model. And the reasons for the existence of short-term memory model deserve further discussion.

\section{Data Availability}

The data are from China Statistical Yearbook, website of China's national bureau of statistics, http://www.stats.gov.cn/,
Guangdong Statistical Yearbook, and Guangdong Statistical Information Network, http:/www.gdstats.gov.cn/tjsj/ gmjjhs/.

\section{Conflicts of Interest}

The authors declare that they have no conflicts of interest.

\section{Acknowledgments}

This work was supported by the National Natural Science Foundation of China (71871084), 2016 Shanghai Maritime University National Key Project Development Project (A20201161107X), and 2015 Shanghai Maritime University Research Start-Up Funding Project (A15101154505Z). At the same time, the authors would like to acknowledge the support of the project of Chinese postdoctoral science foundation 
(2018M632777) and the training program of youth backbone teacher in institutions and universities in Henan province (2018GGJS115).

\section{References}

[1] J. L. Deng, "Introduction to grey system theory," The Journal of Grey System, vol. 1, no. 1, pp. 1-24, 1989.

[2] J. L. Deng, Grey Prediction and Grey Decision, Huazhong University of Science and Technology Press, Wuhan, China, 2002.

[3] B. Zeng and C. Li, "Improved multi-variable grey forecasting model with a dynamic background-value coefficient and its application," Computers \& Industrial Engineering, vol. 118, pp. 278-290, 2018.

[4] J. Ye, Y. Dang, and B. Li, “Grey-Markov prediction model based on background value optimization and central-point triangular whitenization weight function," Communications in Nonlinear Science and Numerical Simulation, vol. 54, pp. 320-330, 2018.

[5] B. Zeng and S. F. Liu, "A self-adaptive intelligence gray prediction model with the optimal fractional order accumulating operator and its application," Mathematical Methods in the Applied Sciences, vol. 23, no. 1, pp. 7843-7857, 2017.

[6] X. Ma and Z. B. Liu, "The GMC $(1, n)$ model with optimized parameters and its application," The Journal of Grey System, vol. 29, no. 4, pp. 122-138, 2017.

[7] P.-H. Hsin and C.-I. Chen, "Application of game theory on parameter optimization of the novel two-stage Nash nonlinear grey Bernoulli model," Communications in Nonlinear Science and Numerical Simulation, vol. 27, no. 1-3, pp. 168-174, 2015.

[8] B. Wei, N. M. Xie, and A. Q. Hu, "Optimal solution for novel grey polynomial prediction model," Applied Mathematical Modelling, vol. 62, pp. 717-727, 2018.

[9] X. Ma, M. Xie, W. Q. Wu, B. Zeng, Y. Wang, and X.-X. Wu, “The novel fractional discrete multivariate grey system model and its application," Applied Mathematical Modelling, vol. 70, pp. 402424, 2019.

[10] L. F. Wu and Z. Y. Zhang, "Grey multivariable convolution model with new information priority accumu lation," Applied Mathematical Modelling, vol. 62, pp. 595-604, 2018.

[11] B. Zeng, H. M. Duan, B. Yun, and M. Wei, "Forecasting the output of shale gas in China using an unbiased grey model and weakening buffer operator," Energy, vol. 151, pp. 238-249, 2018.

[12] X. Ma and Z. B. Liu, "Application of a novel time-delayed polynomial grey model to predict the natural gas consumption in China," Journal of Computational and Applied Mathematics, vol. 324, pp. 17-24, 2017.

[13] Z. X. Wang, Q. Li, and L. L. Pei, "A seasonal GM(1,1) model for forecasting the electricity consumption of the primary economic sectors," Energy, vol. 154, pp. 522-534, 2018.

[14] B. Zeng, Y. T. Tan, H. Xu, J. Quan, L. Y. Wang, and X. Y. Zhou, "Forecasting the electricity consumption of commercial sector in Hong Kong using a novel grey dynamic prediction model," Journal of Grey System, vol. 30, no. 1, pp. 157-172, 2018.

[15] C. Hamzacebi and H. A. Es, "Forecasting the annual electricity consumption of Turkey using an optimized grey model," Energy, vol. 70, no. 3, pp. 165-171, 2014.

[16] D. C. Li, C. J. Chang, C. C. Chen, and W. C. Chen, "Forecasting short-term electricity consumption using the adaptive greybased approach-an Asian case," Omega, vol. 40, no. 6, pp. 767773, 2012
[17] L. F. Wu and H. Y. Zhao, "Using FGM(1,1) model to predict the number of the lightly polluted day in Jing-Jin-Ji region of China," Atmospheric Pollution Research, vol. 10, no. 2, pp. 552$555,2019$.

[18] Z. X. Wang and Q. Li, "Modelling the nonlinear relationship between $\mathrm{CO} 2$ emissions and economic growth using a PSO algorithm-based grey Verhulst model," Journal of Cleaner Production, vol. 207, pp. 214-224, 2019.

[19] W. Meng, D. L. Yang, and H. Huang, "Prediction of China's sulfur dioxide emissions by discrete grey model with fractional order generation operators," Complexity, vol. 2018, no. 1, Article ID 8610679, 13 pages, 2018.

[20] L. F. Wu, N. Li, and Y. J. Yang, "Prediction of air quality indicators for the Beijing-Tianjin-Hebei region," Journal of Cleaner Production, vol. 196, pp. 682-687, 2018.

[21] C.-H. Wang and L.-C. Hsu, "Using genetic algorithms grey theory to forecast high technology industrial output," Applied Mathematics and Computation, vol. 195, no. 1, pp. 256-263, 2008.

[22] Z. Zhao, J. Wang, J. Zhao, and Z. Su, "Using a Grey model optimized by Differential Evolution algorithm to forecast the per capita annual net income of rural households in China," Omega , vol. 40, no. 5, pp. 525-532, 2012.

[23] A. Bezuglov and G. Comert, "Short-term freeway traffic parameter prediction: Application of grey system theory models," Expert Systems with Applications, vol. 62, pp. 284-292, 2016.

[24] X. P. Xiao, J. W. Yang, S. H. Mao, and J. H. Wen, "An improved seasonal rolling grey forecasting model using a cycle truncation accumulated generating operation for traffic flow," Applied Mathematical Modelling, vol. 51, pp. 386-404, 2017.

[25] Y. G. Dang, S. F. Liu, and K. J. Chen, “The GM models that be taken as initial value," Kybernetes, vol. 33, no. 2, pp. 247-254, 2004.

[26] J. Cui, S. F. Liu, B. Zeng, and N. M. Xie, "A novel grey forecasting model and its optimization," Applied Mathematical Modelling, vol. 37, no. 9, pp. 4399-4406, 2013.

[27] W. Zhou and J. M. He, "Generalized GM $(1,1)$ model and its application in forecasting of fuel production," Applied Mathematical Modelling, vol. 37, no. 9, pp. 6234-6243, 2013.

[28] C. I. Chen and S. J. Huang, "The necessary and sufficient condition for $\operatorname{GM}(1,1)$ grey prediction model," Applied and Computational Mathematics, vol. 219, no. 11, pp. 6152-6162, 2013.

[29] G.-D. Li, S. Masuda, D. Yamaguchi, and M. Nagai, "A new reliability prediction model in manufacturing systems," IEEE Transactions on Reliability, vol. 59, no. 1, pp. 170-177, 2010.

[30] N. M. Xie and S. F. Liu, "Discrete grey forecasting model and its optimization," Applied Mathematical Modelling, vol. 33, no. 2, pp. 1173-1186, 2009.

[31] L. F. Wu, S. F. Liu, and L. G. Yao, "Discrete grey model based on fractional order accumulate," System Engineering-Theory \& Practice, vol. 34, no. 7, pp. 1822-1827, 2014.

[32] L. F. Wu, S. F. Liu, L. G. Yao, S. L. Yan, and D. L. Liu, "Grey system model with the fractional order accumulation," Communications in Nonlinear Science and Numerical Simulation, vol.18, no. 7, pp. 1775-1785, 2013.

[33] L. F. Wu, S. F. Liu, W. Cui, D. L. Liu, and T. X. Yao, "Nonhomogenous discrete grey model with fractional-order accumulation," Neural Computing and Applications, vol. 25, no. 5, pp. 1215-1221, 2014.

[34] J. F. Liu, S. F. Liu, L. F. Wu, and Z. G. Fang, "Fractional order reverse accumulative discrete grey model and its application," 
Systems Engineering and Electronics, vol. 38, no. 3, pp. 720-724, 2016.

[35] K. Zhang and S. F. Liu, "Linear time-varying parameters discrete grey forecasting model," Systems Engineering-Theory \& Practice, vol. 30, no. 9, pp. 1650-1657, 2010.

[36] G. W. Stewart, "On the perturbation of pseudo-inverses, projections and linear least squares problems," SIAM Review, vol. 19, no. 4, pp. 634-662, 1977.

[37] J. G. Sun, Matrix Disturbance Analysis, Science Press, Beijing, China, 1987.

[38] L. Zeng, "A new time-varying parameter grey model and its application," Journal of Systems Science and Mathematical Sciences, vol. 37, no. 1, pp. 143-154, 2017. 


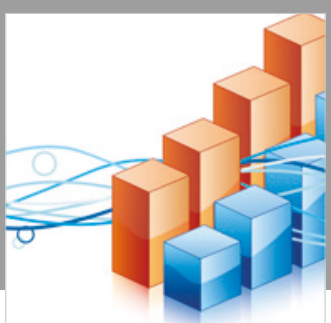

Advances in

Operations Research

\section{-n-m}
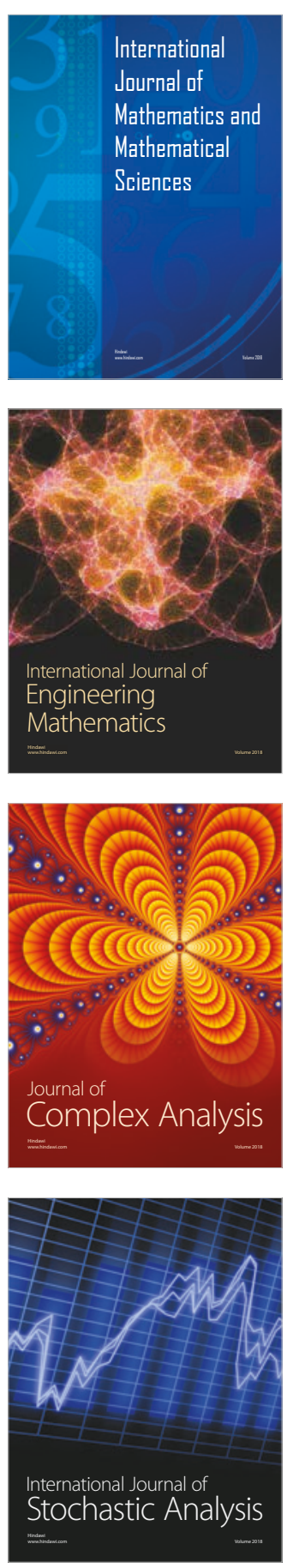
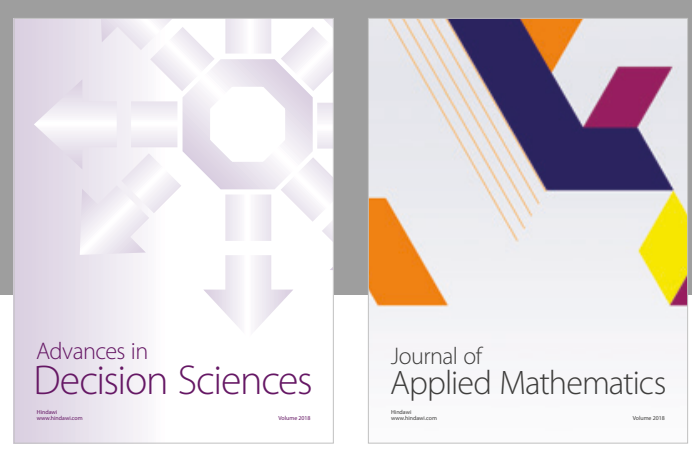

Journal of

Applied Mathematics
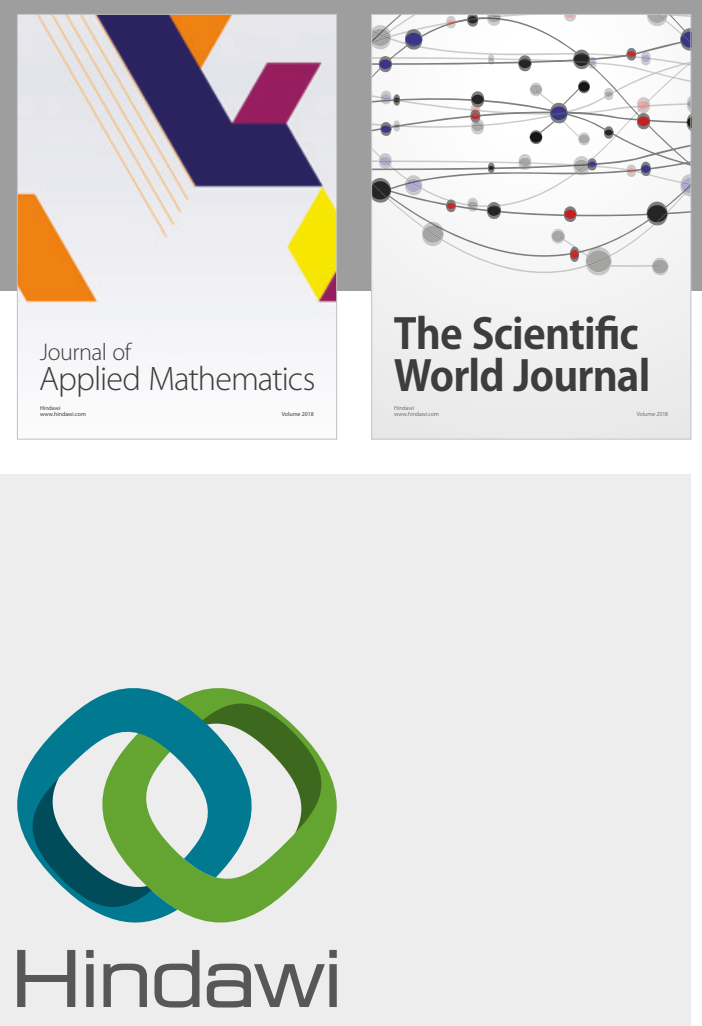

Submit your manuscripts at

www.hindawi.com

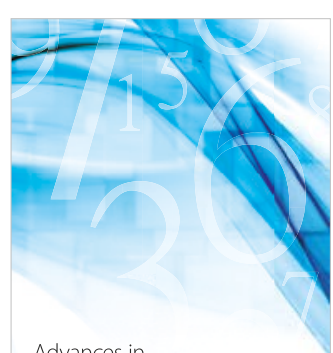

Advances in
Numerical Analysis
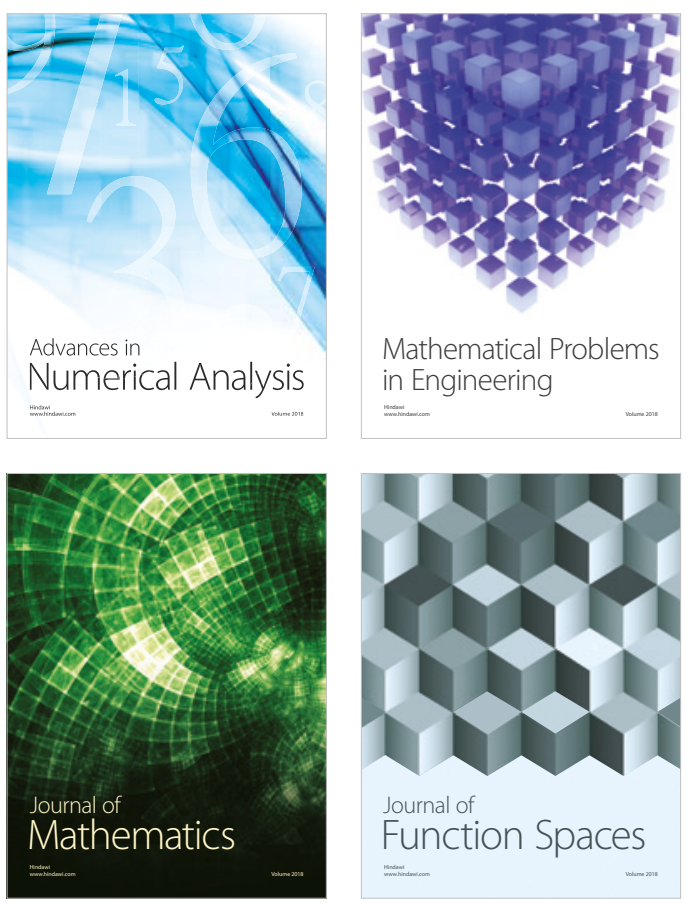

Mathematical Problems in Engineering

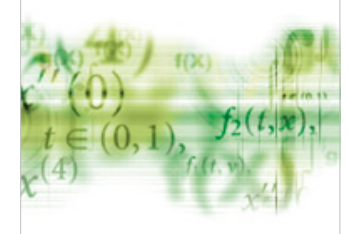

International Journal of

Differential Equations

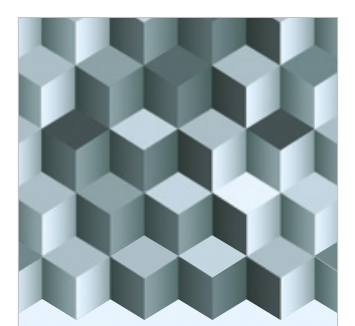

Journal of

Function Spaces

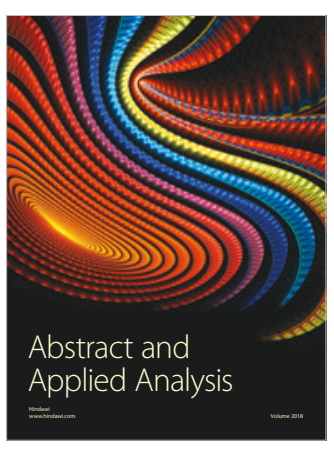

The Scientific

World Journal

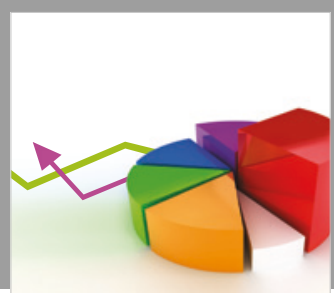

Journal of

Probability and Statistics
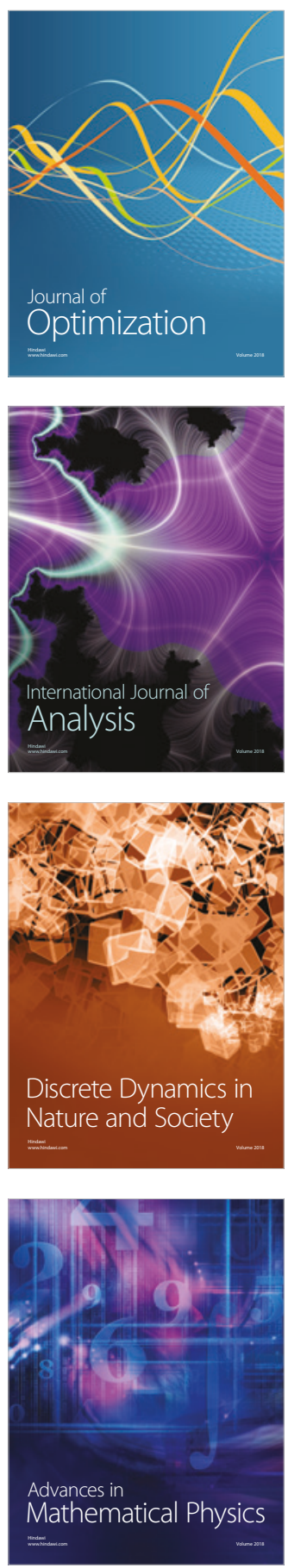